\title{
Nota sobre o Topazio de Arassuaí, Minas Gerais
}

\author{
J. P. Silva \\ (1 figura no texto)
}

O topázio ocorre no Brasil principalmente nos Estados de Minas Gerais e Baía, em zonas distintas: no primeiro deles, ao norte, na bacia do rio Jequitinhonha, encontram-se com frequencia cristais biterminados.

Os cristais que ocorrem em Arassuaí, Teófilo Otoni e Salinas, são geralmente incolôres, azúis ou leves matizes verdes, chegando alguns exemplares a pesar mais de cem quilogramas. Na zona central de Minas Gerais, compreendida entre Ouro Preto e D. Bosco, os cristais, geralmente de dimensões reduzidas, apresentam coloraç̃es que variam do amarelo ao amarelo-conhaque, sendo muito raros, entretanto, os exemplares biterminados.

Na Baía, em Conquista, observam-se belos cristais policróicos, característicos da região.

Segundo Eschwege (1) o topázio ocorre no Brasil em um xisto untuôso e decomposto, de naturêsa talcosa, imerso em xisto argiloso de formação primaria. Já H. GoRCEIX (2). que visitou a região de Ouro Preto, discorda das conclusões de EschWEGE quanto á naturêsa do xisto decomposto, atribuindo-lhe, devido ao seu alto teor em álcalis, um caráter micaceo: esclarece que a este xisto é atribuida a designação de "piçarra" e refere-se á presença constante da litomarga, em filetes brancos, acompanhada quasi 25,35 .

(1) e (4) The American Joumal of Science - XI - 1901 Art. II -

(2) Anais da Escola de Minas de Ouro Preto - I - 1881 - 15, 31. 
sempre de topázios, e, mais raramente, de euclásios. As observações de Djalma Guimarães confirmam suas conclusões (3).

Nota ainda aquele autor que é raro encontrar-se cristais de topázio isolados, sem litomarga, imersos em argila parda, que é o produto de alteração do xisto micaceo. As observações de GoRCEIX se estenderam a toda a região de Ouro Preto, na qual estudou as lavras de Capão-do-Lana, José-Corrêa, Bôa-Vista e Fundão, tendo visto confirmadas suas observações.

ORVILle A. DeRBY (4), após concordar com GorceiX quanto á naturêsa do xisto micaceo, tenta esclarecer, pelos caracteres químicos e mineralógicos dos xistos da região, que muitos deles são provavelmente originários da alteração de rochas eruptivas, e sugeriu a hipótese da alteração anterior ao metamorfismo: entretanto, o argumento em favor da origem eruptiva foi prejudicado pelo fato de se encontrarem em uma rocha similar, embora mais quartzosa, cristais de zircônio de origem clástica.

Estendendo suas observações a outras regiões de Minas, DERBY aponta o grupo dos sienitos-nefelínicos como o mais provavel típo de rocha originária do xisto, e o justifica mostrando que estas rochas são mais particularmente sujeitas a alterações, dando massas segregadas, chamadas "schlieren"

E' interessante notar que o estudo dos xistos mais normais dessa região, bem como de Diamantina, conduziram ás mesmas conclusões: as rochas do grupo dos sienitos-nefelínicos estão alí representadas ao lado de rochas de origem clástica. Nota ainda O. Derby que, embora não seja recordada a presença do topázio nas rochas do típo alí mencionados, não ha razão aparente pela qual não poderia ser encontrado, visto que o único elemento requerido para a sua formação é o fluor, que, sob a forma de fluorita e fluosilicatos, é muito freqüente nas suas segregações e cavidades drúsicas.

Contudo, aquele autor esclarece que a rocha originária do xisto dificilmente poderia ser identificada de modo positivo, observando-se que a questão da gênese do topázio de Ouro Preto, em particular, parece estar longe de esclarecer-nos completamente.

Com relação ao municipio de Arassuaí, o topázio ocorre, segundo OTHON H. LEONARDOS (5) em veios de pegmatito, os quais afloram, em Córrego das Neves, em diques no gnais. Citam-se casos em que o topázio é encontrado em rochas do grupo dos riolitos, como se observa nas regiões de Utah e Colorado, EE. UU. (6), onde parece resultar de ações pneumatolíticas.

(3) Djalma Guimarães - Anais da Academia Brasileira de Ciências.

(5) Othon H. Leonardos - Tantalo, Niobio, Uranio e Radio no Brasil, D.N.P.M. Bol. II -1936.

(6) A Textbook of Mineralogy - E. S. DANA - $1932-543$. 
Acompanham-no, ás vezes, a cassiterita, a fluorita e a turmalina, sendo que no Brasil a associação á primeira é menos frequente, contrariamente ao que se observa em Jachymov, no Erzebirge, Boêmia: a associação á fluorita é frequente na região de South Carolina, EE. UU., ocorrendo contudo juntamente com o berilo nos pegmatitos, em Figueira, a leste de Minas Gerais.

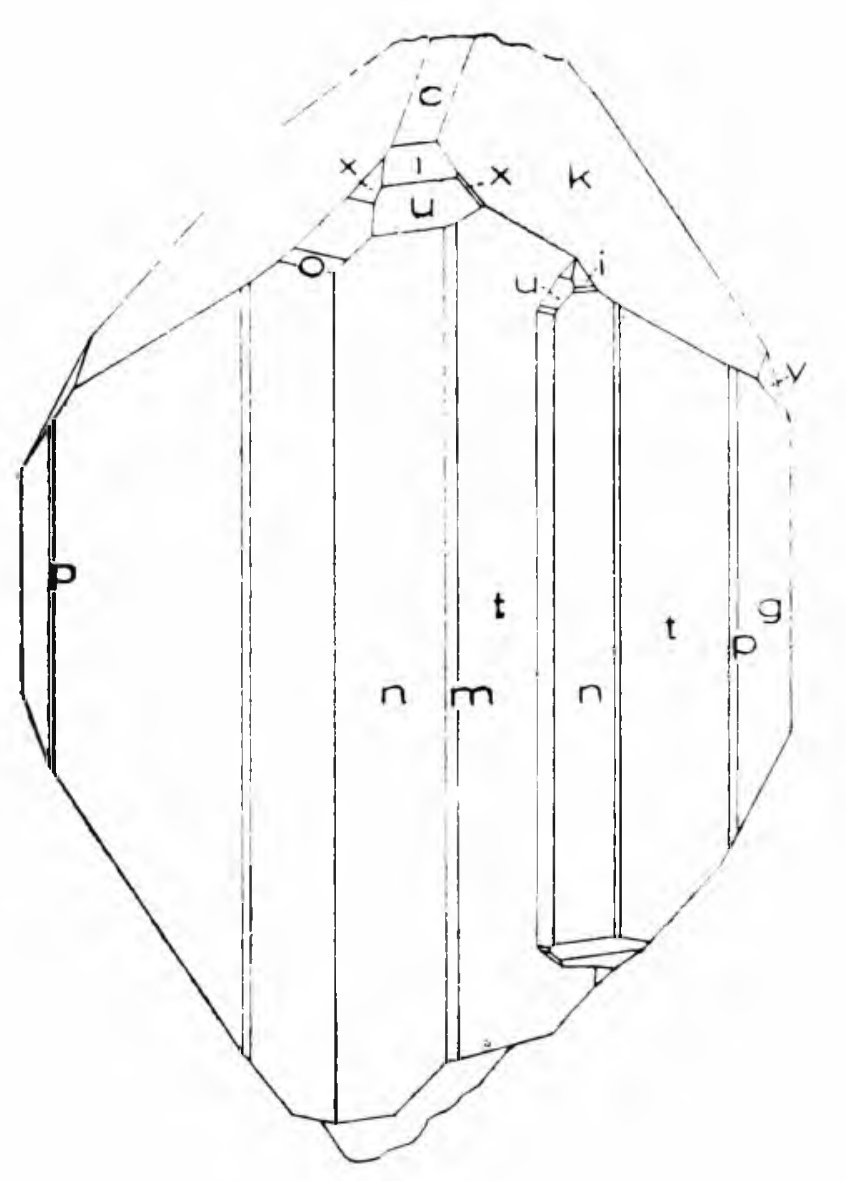

Fig. 1

O cristal, pertencente á coleção Araujo Ferraz do Museu da Faculdade de Filosofia, Ciências e Letras de São Paulo, que por designação do sr. Prof. E. OnORato, tivemos ocasião de estudar, é, pela sua grandesa e característicos morfológicos, um raro exemplar de topázio do Brasil (fig. 1). E' dotado de côr verde-azulada e perfeita limpidez, oferecendo as faces, bastante polidas, belos refléxos. Apresenta nitida biterminação, com perfeito pinacóide basal, bem como destacado acrecimento paralélo.

Mede o exemplar $47,8 \mathrm{~cm}$. na direção $z, 32,3 \mathrm{~cm}$. e $37,0 \mathrm{~cm}$. nas direções $\mathrm{x}$ e $\mathrm{y}$. respectivamente: nêle notamse leves estrías nas faces dos prismas verticais, bem como

planos de clivagem interna segundo (001)

As formas encontradas, em número de doze, são as seguintes:

$$
\begin{aligned}
& \mathrm{n}\{110\} \mathrm{m}\{230\} \mathrm{p}\{250\} \mathrm{t}\{120\} \mathrm{g}\{130\} \\
& \mathrm{k}\{011\} \quad \mathrm{y}\{021\} \mathrm{c}\{001\} \\
& \text { o }\{111\} \quad \text { u }\{112\} \quad \mathrm{i}\{113\} \quad \mathrm{x}\{123\}
\end{aligned}
$$

O habitus é o prismático vertical, apresentando o cristal numerosas faces na zona [001], todas alongadas na direção $\mathrm{z}$.

Nessa zona sobresáe a forma $\{120\}$ pelo seu desenvolvimento, como a mais importante do cristal, apresentando as faces (120) e (120) perturbações de crescimento e leves estrías verticais. Os prismas $\{130\}$ e $\{110\}$ atingem menor desenvolvimento, sendo 
contudo de certa importancia. As demais formas da zona [001] apresentam-se muito estreitas, e igualmente alongadas.

$\mathrm{Na}$ zona [100] são mais importantes pela sua estensão as faces da forma $\{011\}$, notando-se entre elas a face (011), com imperfeições no seu crescimento. São regulares e perfeitamente polidas as faces de $\{021\}$, bem como o pinacoide $\{001\}$, alongado na direção $\mathrm{x}$.

As demais faces do cristal são de menores dimensões e perfeitamente polidas.

Notamos que as zonas prismáticas vertical e horizontal [100], caracterisam a conformação mais frequente do topázio das Américas. De fato, PEACock (7), estudando duas desênas de cristais de Devil's Head, Colorado, acusa sua freqüencia e predominancia. Os exemplares dessa região são, em relação ao que foi por nós estudado, mais ricos em formas, apresentando-as mais numerosas e complexas, como $\{103\}$ e $\{023\}$

Com as medidas dos angulos $(001):(011)$ e (100):(110) calculamos a seguinte relação paramétrica:

$$
a: b: c=0,52854: 1: 0,95423
$$

Estas constantes têm valores próximos aos encontrados por Peacock.

$$
\mathrm{a}: \mathrm{b}: \mathrm{c}=0,5285: 1: 0,9540
$$

bem como ans valores para os elementos de Kokscharow.

$$
\text { a }: b: c=0,52854: 1: 0,95395
$$

Apresentamos a seguir a tabela dos angulos calculados, cujos valores se aproximam dos resultados das medidas.

(7) The American Mineralogist - V $20-1935-\mathrm{n} .^{\circ} 5-354$.

(8) Idem (7).

(9) DöELTER - Handbuch $-2 \mathrm{Bd}-2$ Hälfte. 
Nota sobre um topazio de Arassuai

\begin{tabular}{|c|c|c|c|c|c|}
\hline \multirow{2}{*}{$\begin{array}{l}\text { Angulos } \\
\text { medidos }\end{array}$} & \multirow{2}{*}{$\begin{array}{l}\text { Num. } \\
\text { med. }\end{array}$} & \multicolumn{2}{|c|}{ V A L O R E S } & \multirow{2}{*}{$\begin{array}{l}\text { Valores } \\
\text { médios }\end{array}$} & \multirow{2}{*}{$\begin{array}{c}\text { Valores } \\
\text { calculados }\end{array}$} \\
\hline & & minimos & maximos & & \\
\hline $110-110$ & 5 & $55^{\circ} 43^{\prime}$ & $55^{\circ} \stackrel{1}{\prime} 4^{\prime}$ & $55^{\circ} 43^{1 / 4}$ & $55^{\circ} 42^{\prime} 53^{\prime \prime}$ \\
\hline $110-230$ & 13 & $10^{\circ} 23^{\prime}$ & $10^{\circ} 27^{\prime} 1 / 2$ & $10^{\circ} 24^{1 / 2}$ & $10^{\circ} 33^{\prime} 1 / 2$ \\
\hline $120-230$ & 13 & $8^{\circ} 16^{\prime}$ & $8^{2} 19^{\prime}$ & $8^{c} 17^{1 / 2}$ & $8^{\circ} 10^{\prime} 50^{\prime \prime}$ \\
\hline $120-250$ & 13 & $5^{\circ} 531 / 2{ }^{\prime}$ & $6^{\circ} \quad 9^{\prime}$ & $6^{\circ} \quad 6^{\prime} 40$ & $6^{\circ} 17^{\prime} 1 / 2$ \\
\hline $130-250$ & 10 & $4^{\circ} 37^{\prime}$ & $4^{\circ} 58^{\prime}$ & $40541 / z$ & $4^{\circ} 52^{\prime} 50^{\prime \prime}$ \\
\hline $001-011$ & 11 & $43^{\circ} 38^{\prime}$ & $43^{\circ} 41^{\prime}$ & $43^{\circ} 40^{\prime}$ & $43^{\circ} 39^{\prime} 1 / 2$ \\
\hline 011-021 & 11 & $18^{\circ} 30^{\prime}$ & $18^{\circ} 42^{\prime}$ & $18^{\circ} 36^{\prime}$ & $18^{\circ} 41^{\prime} 20^{\prime \prime}$ \\
\hline $130-\overline{130}$ & 8 & $64^{\circ} 28^{\prime}$ & $64^{\circ} 29^{\prime}$ & $64^{\circ} 28^{1 / 2}$ & $64^{\circ} 28^{\prime}$ \\
\hline $110-112$ & 12 & $44^{\circ} 28^{\prime}$ & $44^{\circ} 31 \frac{1 / 2^{\prime}}{}$ & $44^{\circ} 29^{\prime}$ & $44^{\circ} 24^{\prime} 15^{\prime \prime}$ \\
\hline $110-112$ & 7 & $66^{\circ} \quad 8^{\prime}$ & $66^{\circ} 14^{\prime}$ & $66^{\circ} 11^{\prime}$ & $66^{\circ} 16^{\prime} 10^{\prime \prime}$ \\
\hline $130-021$ & 15 & $41^{\circ} 27^{1 / 2}$ & $41^{\circ} 29^{\prime}$ & $41^{\circ} 281 / z^{\prime}$ & $41^{\circ} 28^{\prime} 40^{\prime \prime}$ \\
\hline $110-011$ & 12 & $71^{\circ} 11^{\prime}$ & $71^{\circ} 15^{\prime}$ & $71^{\circ} 13^{\prime} 40^{\prime \prime}$ & $71^{\circ} 10^{\prime} 50^{\prime \prime}$ \\
\hline 011-112 & 8 & $42^{\circ} 24^{\prime}$ & $42^{\circ} 35^{\prime}$ & $42^{\circ} 31^{\prime} 40^{\prime \prime}$ & $42^{\circ} 33^{\prime}$ \\
\hline 011-113 & $1: 3$ & $38^{\circ} 46^{1 / 2}$ & $38^{\circ} 52^{\prime}$ & $38^{\circ} 47^{\prime} 50^{\prime \prime}$ & $38^{\circ} 46^{\prime} 38^{\prime \prime}$ \\
\hline $1 \overline{10}-1 \overline{11}$ & 5 & $26^{\circ} \quad 5^{\prime}$ & $26^{\circ} 13^{\prime}$ & $26^{\circ} \quad 9^{\prime}$ & $26^{\circ} \quad 5^{\prime} 30^{\prime \prime}$ \\
\hline 001-113 & 8 & $34^{\circ} \quad 4^{\prime}$ & $34^{\circ} 17^{\prime}$ & $34^{\circ} 13^{\prime}$ & $34^{\circ} 14^{\prime} 1 / 2$ \\
\hline $120-011$ & 20 & $59^{\circ} 52^{\prime}$ & $60^{\circ} \quad 3^{\prime}$ & $59^{\circ} 56^{\prime} 36^{\prime \prime}$ & $59^{\circ} 54^{\prime} 1 / 2$ \\
\hline $120-112$ & 5 & $47^{\circ} 26^{\prime}$ & $47^{\circ} 29^{1 / 2^{\prime}}$ & $47^{\circ} 27^{\prime} 40^{\prime \prime}$ & $47^{\circ} 25^{\prime} 20^{\prime \prime}$ \\
\hline $130-011$ & 6 & $54^{\circ} 14^{1 / 2} 2^{\prime}$ & $54^{\circ} 17^{1 / 2^{\prime}}$ & $54^{\circ} 16^{\prime}$ & $54^{\circ} 16^{\prime} 20^{\prime \prime}$ \\
\hline$\overline{112}-112$ & 2 & 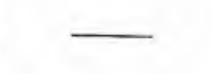 & - & $39^{\circ} \quad 5^{\prime}$ & $39^{\circ} \quad 2^{\prime}$ \\
\hline$\overline{111}-1 \overline{12}$ & 6 & $18^{\circ} 17^{\prime}$ & $18^{\circ} 27^{\prime}$ & $18^{\circ} 22^{\prime}$ & $18^{\circ} 18^{\prime} 50^{\prime \prime}$ \\
\hline $112-113$ & 17 & $11^{\circ} \quad 6^{\prime}$ & $11^{\circ} 23^{\prime}$ & $11^{\circ} 181 / 2^{\prime}$ & $11^{\circ} 21^{\prime}$ \\
\hline $112-1 \overline{13}$ & 6 & $36^{\circ} 20^{\prime}$ & $36^{\circ} 27^{\prime}$ & $36^{\circ} 231 / 2^{\prime}$ & $36^{\circ} 24^{\prime} 10^{\prime \prime}$ \\
\hline $112-123$ & 3 & $\longrightarrow$ & - & $13^{\circ} 27^{\prime}$ & $13^{\circ} 33^{\prime} 20^{\prime \prime}$ \\
\hline $011-123$ & 2 & $\longrightarrow$ & $\longrightarrow$ & $28^{\circ} 58^{1 / 2}$ & $28^{\circ} 59^{\prime} 10^{\prime \prime}$ \\
\hline$\overline{0} \overline{11}-2 \overline{30}$ & 7 & $64^{\circ} 26^{\prime}$ & $64^{\circ} 35^{1 / 2}$ & $64^{\circ} 291 / 2^{\prime}$ & $64^{\circ} 30^{\prime}$ \\
\hline $0 \overline{1} 1-113$ & 3 & $65^{\circ} 15^{1 / 2}$ & $65^{\circ} 20^{1 / 2^{\prime}}$ & $65^{\circ} 18^{\prime}$ & $65^{\circ} 23^{\prime}$ \\
\hline
\end{tabular}





\section{SUMMARIUM}

\section{EX ARASSUAÍ (MINAS GERAIS) TOPAZII EXPEDITIO}

Qui reperiuntur topazii in Arassuaí, ii plerumque incolores, caerulei aut viridi lenitate colorati sunt. Deprehenduntur pegmatiti in venis quae subeunt in collectionis gneissi.

Quod scrutati sumus crystallum id ob rarum magnitudinem et insignes morphologicos conspicitur. Biterminatum, colore glacum et limpidum.

Habitus prismaticus verticalis. Erant ei amplitudines : $47,8 \mathrm{~mm}$ ad $z: 32,3 \mathrm{~mm}$. ad $x ; 37,0 \mathrm{~mm}$. ad $y$.

Quas reperimus formas:

$$
\begin{aligned}
& \mathrm{n}\{110\}, \mathrm{m}\{230\}, \mathrm{p}\{250\}, \mathrm{t}\{120\}, \mathrm{g}\{130\} \\
& \mathrm{k}\{011\}, \mathrm{y}\{021\}, \mathrm{c}\{001\} \\
& \mathrm{o}\{111\}, \mathrm{u}\{112\}, \mathrm{i}\{113\}, \mathrm{x}\{123\}
\end{aligned}
$$

Constans: $\quad \mathrm{a}: \mathrm{b}: \mathrm{c}=0,52854: 1: 0,95423$ 